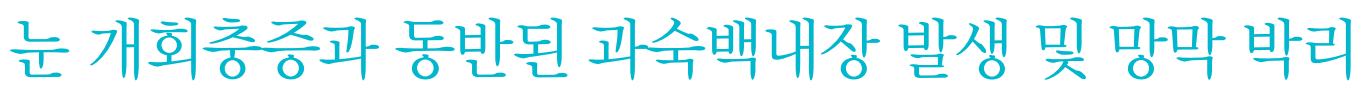

\title{
Ocular Toxocariasis Combined with Hypermature Cataract Formation and Retinal Detachment
}

\author{
장철원, 김정희, 황덕진 \\ Cheol Won Jang, Jeong Hee Kim, Duck Jin Hwang \\ 한길안과병원 \\ Hangil Eye Hospital, Incheon, Korea
}

Purpose: To describe a case of ocular toxocariasis combined with hypermature cataract formation and retinal detachment.

Case summary: A 47-year-old man with decreased visual acuity in his left eye was diagnosed with white cataract and retinal detachment. His initial best corrected distance visual acuity (BCVA) was light perception in the left eye, and he underwent vitrectomy for the retinal detachment. After 4 days, the fundus examination revealed a flat posterior pole, but the serology test showed positive results for the $\lg \mathrm{G}$ antibody of toxocariasis. For toxocariasis treatment, albendazole was prescribed for 2 weeks; after 1-year of follow up, the BCVA had improved to counting fingers at $20 \mathrm{~cm}$.

Conclusions: We report a case of ocular toxocariasis combined with hypermature cataract formation and retinal detachment. Ocular toxocariasis is a possible cause of retinal detachment combined with hypermature cataract.

Keywords: Hypermature cataract; Retinal detachment; Toxocariasis

\section{Introduction}

Ocular toxocariasis is a zoonotic disease caused by either Toxocara canis or Toxocara cati larvae [1-5]. Due to inflammation, the most common symptoms of ocular toxocariasis are blurred vision and floaters [1-4]. Granulomas in both the posterior pole and the periphery are common characteristics in ocular toxocariasis [1-4]. Other clinical manifestations are tractional retinal detachment, retinal hemorrhage, and macular edema [1-4]. Cataract is another clinical finding in ocular toxocariasis, the most common type being a posterior subcapsular cataract showing granuloma-like opacity [5]. To our knowledge, this is the first report of a patient with a hypermature cataract and a retinal detachment, both of which may have been be caused by ocular toxocariasis. Here in, we describe the clinical features of a patient with a hypermature cataract diagnosed with ocular toxocariasis after surgery for a retinal detachment.

\section{Case Report}

A 47-year-old man presented with complaints of decreased visual acuity in his left eye over the preceding few months.
Address reprint requests to Duck Jin Hwang, MD

Hangil Eye Hospital, \#35 Bupyeong-daero, Bupyeong-gu, Incheon 21388, Korea Tel: 82- 32-503-3322, Fax: 82-32-504-3322

E-mail: hallelu7@gmail.com
Received: 2016. 10. 17

Revised: 2016. 11. 1

Accepted: 2016. 11. 2 


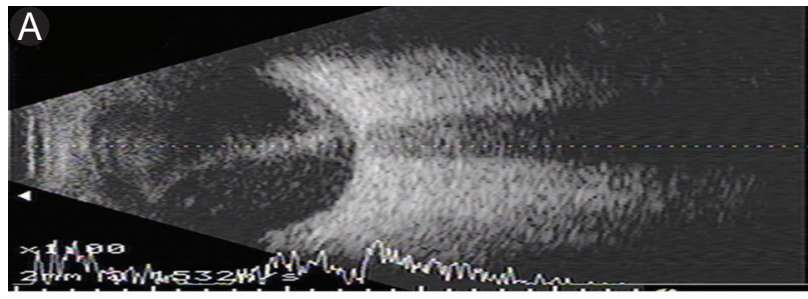

B
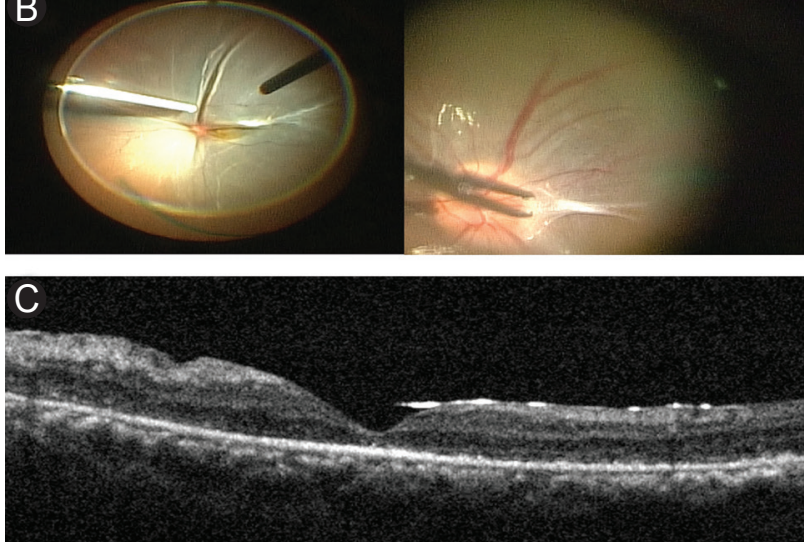

Figure 1. Preoperative, intraoperative and postoperative images. (A) B-scan showing retinal detachment in the left eye. (B) A fibrous band from the macula, connected to the optic disc, was observed during vitrectomy after removing the vitreous opacity. (C) Spectral domain optical coherence tomography showed a flat macula on postoperative day 4.

The patient had a 10-year history of type II diabetes and was receiving insulin treatment. He also had chronic hepatitis B with liver cirrhosis, for which he was receiving treatment. He denied having traveled out of the country and having had contact with animals or animal feces. His initial best corrected distance visual acuity (BCVA) was 20/25 in the right eye, with only light perception in the left eye. Slit lamp examination showed mild nuclear lens opacity in the right eye and a white cataract in the left eye. There was no sign of inflammation in the anterior segment of either eye. Because of the white cataract, it was not possible to examine the fundus of the left eye. A B-scan of the left eye revealed retinal detachment (Fig. 1A). The fundus examination of the right eye was unremarkable with no diabetic retinopathy. During cataract surgery and vitrectomy for the retinal detachment, proliferative vitreoretinopathy and vitreous opacity with fibrous bands were also observed (Fig. 1B). Infectious uveitis was suspected, and a silicone oil injection was performed. Neither diabetic retinopathy nor peripheral granuloma was observed. On postoperative day 4, spectral domain optical coherence tomography showed a flat macula and peripheral retina (Fig. 1C). The serology test showed positive results for the IgG antibody of toxocariasis. For toxocariasis treatment, albendazole $800 \mathrm{mg}$ b.i.d. was prescribed for 2 weeks. At the 1-year follow up, a fundus examination with silicone oil showed a flat macula and peripheral retina, and the BCVA had improved to counting fingers at $20 \mathrm{~cm}$

\section{Discussion}

Ocular toxocariasis is an uncommon disease with a prevalence ranging from $0 \%$ to $3.1 \%$ [4]. Stewart et al. [4] described the most common clinical signs to be vitreous inflammation, cystoid macular edema, vitreoretinal traction strands leading to the optic disc, and/or a granuloma. They concluded that, of all clinical features of ocular toxocariasis, vitritis was the most common cause of vision loss. To our knowledge, no previous studies have reported an incidence of a hypermature cataract combined with ocular toxocariasis. Ahn et al. [5] found that all of the 7 patients in their case series showed granuloma-like lens opacities that closely resembled retinal granulomas in appearance in the eye affected by ocular toxocariasis. They concluded that the most common type of cataract associated with ocular toxocariasis was posterior subcapsular. In all cases, peripheral granuloma was found, and its presence seemed to be associated with the formation of granuloma-like cataracts. While visual axis opacification was uncommon in their case series, in our case, it was severely affected by both the hypermature cataract and retinal detachment.

Further, both proliferative vitreoretinopathy and vitreous opacity with a fibrous band indicated chronicity and might have caused total retinal detachment. The vitreous opacity may have been a result of the ocular toxocariasis. However, it is possible that vitreous opacity was not associated with the ocular toxocariasis, because the causal relationship between ocular toxocariasis and cataracts or retinal detachment is not clear. Both the vitreous opacity and the fibrotic retinal band were masked by a hypermature cataract in the slit lamp examination. In the absence of any clinical signs of ocular toxocariasis, a serum enzyme-linked immunosorbent assay for antibodies to Toxocara was helpful in diagnosing ocular toxocariasis in this case. Ocular toxocariasis might be a cause of retinal detachment combined with hypermature cataract. Further careful observation would be helpful 
for a better understanding of ocular toxocariasis and retinal detachment with hypermature cataract, especially due to the rare incidence of these combined diseases.

\section{Conflicts of interest}

The authors report no conflicts of interest. The authors alone are responsible for the content and writing of the paper.

\section{References}

1. Zhou M, Chang Q, Gonzales JA, et al. Clinical characteristics of ocular toxocariasis in Eastern China. Graefes Arch Clin Exp Ophthalmol 2012;250:1373-8.

2. Kwon SI, Lee JP, Park SP, et al. Ocular toxocariasis in Korea. Jpn J Ophthalmol 2011;55:143-7.

3. Acar N, Kapran Z, Utine CA, Büyükbabani N. Pars plana vitrectomy revealed Toxocara canis organism. Int Ophthalmol 2007;27:277-80. Epub 2007 May 8.

4. Stewart JM, Cubillan LD, Cunningham ET Jr. Prevalence, clinical features, and causes of vision loss among patients with ocular toxocariasis. Retina 2005;25:1005-13.

5. Ahn SJ, Woo SJ, Hyon JY, Park KH. Cataract formation associated with ocular toxocariasis. J Cataract Refract Surg 2013;39:830-5. 